\title{
COMPARISONS OF FEC AND CODEC ROBUSTNESS ON VOIP QUALITY AND BANDWIDTH EFFICIENCY
}

\author{
WENYU JIANG AND HENNING SCHULZRINNE \\ Columbia University, Department of Computer Science \\ 1214 Amsterdam Ave, Mail Code 0401, New York, NY 10027, USA \\ Email: \{wenyu,hgs\}@cs.columbia.edu
}

\begin{abstract}
Packet loss and delay in the Internet degrades the quality of Voice over IP (VoIP). Forward error correction (FEC) reduces packet loss at the cost of higher delay and bandwidth. An alternative is a loss-robust voice codec, but also at the expense of higher bit-rates. We compare the perceived quality of both schemes under the same bandwidth requirement. We evaluate two conventional low bit-rate codecs, G.729 and G.723.1, and one robust codec, iLBC. G.729 with FEC generally prevails, but when considering delay impairment, iLBC is better under low packet loss. If iLBC also uses FEC, then its quality can sometimes be better than G.729 plus FEC with the same gross bit-rate. The comparison with G.723.1 is similar, except that G.723.1 is less advantageous for low packet loss because its intrinsic quality is slightly lower than iLBC. Finally, given a target bit-rate, we compute the maximum achievable MOS using any of these codecs. It exhibits an upper limit however large the target bit-rate is, due to the inherent delay impairment of FEC.
\end{abstract}

\section{Introduction}

Voice over IP uses low bit-rate codecs to save on bandwidth and communication costs. However, packet loss can result in lower voice quality. Because the Internet is still a best-effort network without general quality of service support, users must rely on end-to-end techniques to improve quality.

Forward error correction (FEC) ${ }^{1}$ recovers lost packets by transmitting redundant data. A commonly used FEC code is the Reed-Solomon ${ }^{2}$ code. Its notation is $(n, k)$, where $n$ and $k$ are the number of total and payload units in a block, respectively. It can recover the lost packets if and only if at least $k$ out of $n$ packets in a block are received. FEC's main disadvantage is the additional recovery delay and bandwidth overhead. An $(n, k)$ code introduces a delay of $n \cdot T$ (including packetization delay), where $T$ is the packet interval.

Alternatively we can design a more robust codec. It should be frameindependent, as otherwise one frame loss would cause many subsequent voice frames to be decoded incorrectly. The recent iLBC codec ${ }^{3}$ is the only such candidate, ${ }^{a}$ which runs at $14 \mathrm{~kb} / \mathrm{s}$ with a $30 \mathrm{~ms}$ frame length.

${ }^{a}$ G.711 is also frame-independent, but its high bit-rate $(64 \mathrm{~kb} / \mathrm{s})$ makes it less suitable for wide area network telephony, where bandwidth can be scarce during congestion

icn 2002: submitted to World Scientific on June 5, 2002 
Given the common objective of FEC and robust codec design, it is valuable to know which scheme gives better quality. The perceived quality in VoIP is measured by the Mean Opinion Score (MOS). ${ }^{4}$ MOS rates the audio from 5 to 1, with 5 being Excellent, 4 Good, 3 Fair, 2 Poor, and 1 Bad, respectively. MOS is obtained by listening tests, where each listener rates an audio clip from 5 to 1 , and the resulting average is the MOS.

Figure 1(a) shows the MOS performance of iLBC, G.729 ${ }^{5}(8 \mathrm{~kb} / \mathrm{s})$ and G.723.1 ${ }^{6}(6.3 \mathrm{~kb} / \mathrm{s})$ under packet loss. The MOS of iLBC at $15 \%$ loss is close to G.729 under $8 \%$ loss. Such robustness comes at a slightly higher bit-rate, because iLBC encodes every frame independently as opposed to incrementally.

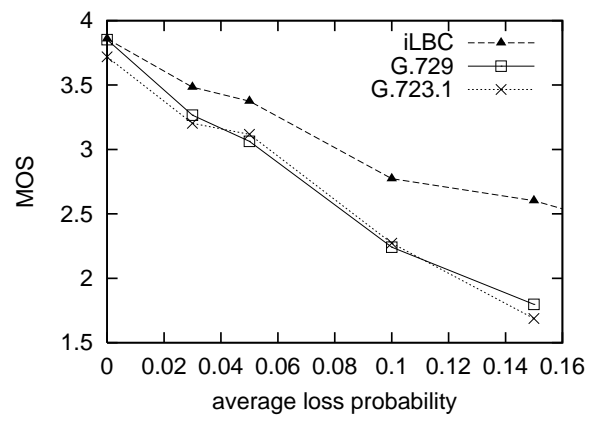

(a) MOS performance without $\mathrm{FEC}^{7}$

\begin{tabular}{|c|l|rrc|}
\hline & codec & $\begin{array}{c}\text { media } \\
\text { pkt_size }\end{array}$ & $r_{\text {media }}$ & \multicolumn{1}{c|}{$r_{I P}$} \\
\hline \multirow{2}{*}{ no } & iLBC & 54 bytes & $14.4 \mathrm{~kb} / \mathrm{s}$ & $25.1 \mathrm{~kb} / \mathrm{s}$ \\
FEC & G.729 & 30 bytes & $8 \mathrm{~kb} / \mathrm{s}$ & $18.7 \mathrm{~kb} / \mathrm{s}$ \\
& G.723.1 & 24 bytes & $6.4 \mathrm{~kb} / \mathrm{s}$ & $17.1 \mathrm{~kb} / \mathrm{s}$ \\
\hline \multirow{2}{*}{$(4,2)$} & iLBC & 108 bytes & $28.8 \mathrm{~kb} / \mathrm{s}$ & $39.5 \mathrm{~kb} / \mathrm{s}$ \\
FEC & G.729 & 60 bytes & $16 \mathrm{~kb} / \mathrm{s}$ & $26.7 \mathrm{~kb} / \mathrm{s}$ \\
& G.723.1 & 48 bytes & $12.8 \mathrm{~kb} / \mathrm{s}$ & $23.5 \mathrm{~kb} / \mathrm{s}$ \\
\hline
\end{tabular}

(b) Bandwidth overhead of FEC

Figure 1. Example codec MOS performance and FEC overhead; packet interval $T=30 \mathrm{~ms}$

The overhead of FEC is not necessarily high once we consider the typical 40 byte IP/UDP/RTP ${ }^{8}$ packet header. Assuming piggy-backing ${ }^{9}$ in FEC, the media and IP bit-rates for an $(n, k)$ code are then calculated as:

$$
r_{\text {media }}=r_{\text {codec }} \cdot \frac{n}{k}, \quad r_{I P}=r_{\text {media }}+\frac{40 b y t e s}{T}
$$

where $r_{\text {codec }}$ is the codec bit-rate, $T$ is the packet interval. Figure 1(b) shows some typical packet sizes and bit-rates FEC introduces.

Our main contributions include an original study on the quality tradeoff between FEC and codec robustness under packet loss with the same bandwidth requirement, evaluating both listening and conversational quality, where delay is an important factor. We also investigate the best quality attainable under packet loss utilizing both schemes within a target bit-rate.

The rest of this paper is organized as follows. Section 2 shows how to predict MOS quality of FEC. Section 3 compares FEC and codec robustness. Section 4 discusses the effect of target bit-rate on achievable quality. Section 5 lists related work and section 6 concludes the paper.

icn 2002: submitted to World Scientific on June 5, 2002 


\section{Prediction of MOS Quality under FEC}

\subsection{The E-model}

MOS listening tests are time consuming. An alternative is the E-model, 10 an analytical model for predicting MOS. Cole and Rosenbluth ${ }^{11}$ discusses its application in a VoIP environment by simplifying the model into two parameters, loss and delay. The procedures are summarized below:

1. Translate loss and delay into impairment scores, denoted as $I_{e}$ and $I_{d}$, respectively, as in Figure 2. Mapping for $I_{e}$ is derived from MOS curves.

2. Compute a score called the $R$ value as follows:

$$
R=94.2-I_{d}-I_{e}
$$

3. Map the $R$ value to MOS with a fixed, reversible mapping function. ${ }^{11}$

$$
M O S=1+0.035 \cdot R+7 \times 10^{-6} \cdot R \cdot(R-60) \cdot(100-R)
$$

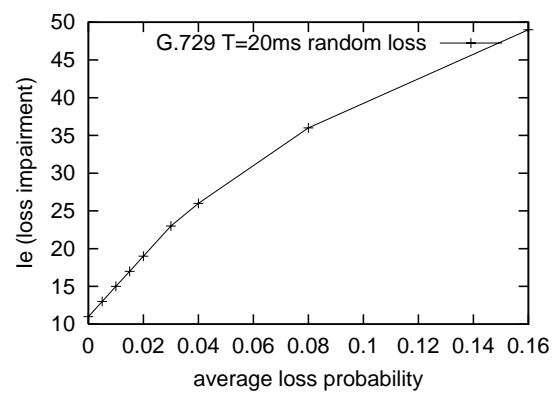

(a) loss impairment $I_{e}$ for G.729 ${ }^{10}$

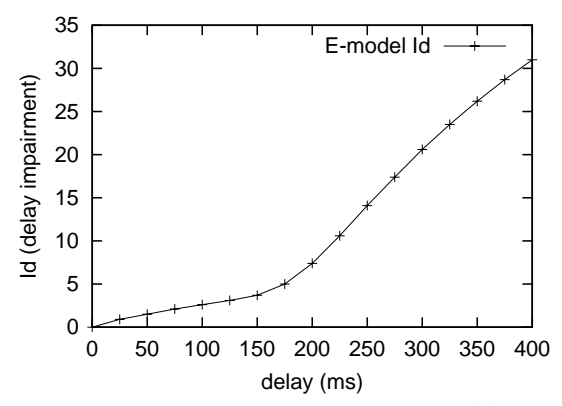

(b) delay impairment $I_{d}{ }^{11}$

Figure 2. Effect of loss and delay in the E-model

In an earlier paper ${ }^{9}$ we present a method for predicting FEC MOS based on the E-model. First we reuse an existing MOS curve (e.g., Figure 1) and reverse map MOS to $R$ value by inverting equation (3). Then $R$ is mapped to $I_{e}$, where $I_{e}=94.2-R$, a reverse application of equation (2). This way we establish a loss to $I_{e}$ mapping just as in Figure 2(a). Second, we compute the final loss probability (denoted as $p_{f}$ ) after applying FEC. The method of calculating $p_{f}$ is either analytical for simple FEC codes, ${ }^{9}$ or by simulation for complex FEC codes. Then, we plug $p_{f}$ into our reverse-engineered loss to $I_{e}$ mapping and obtain $I_{e}$. The delay of an $(n, k)$ code is $n \cdot T$, which is translated into $I_{d}$ using Figure 2(b). From there we can compute the $R$ value and obtain the FEC MOS. We have compared this analytical method with our real FEC MOS test results ${ }^{9}$, and the analytical prediction is fairly accurate. 


\section{Quality Evaluation of FEC vs. Codec Robustness}

We compare iLBC, G.729 and G.723.1 ${ }^{b}$ and our evaluation comprises three steps. First, only G.729 uses FEC. Next, we add FEC to both iLBC and G.729 while keeping the same gross bit-rate between them. Finally, we repeat these steps for G.723.1 and iLBC. We examine both listening MOS and conversational MOS $\left(M O S_{c}\right)$, with the latter considering delay impairment.

Packet loss in the Internet is often bursty, ${ }^{12}$ so we use the Gilbert model ${ }^{9}$ to simulate bursty loss. In our evaluation, loss burstiness, expressed in conditional loss probability, is set to $30 \%$ at $30 \mathrm{~ms}$ packet interval.

\subsection{G.729 with FEC vs. iLBC without FEC}

Figure 3 compares the plain iLBC and G.729 with a $(5,3)$ FEC code. Thus G.729 has a gross bit-rate of $8 \times 5 / 3=13.3 \mathrm{~kb} / \mathrm{s}$, about the same as iLBC $(14 \mathrm{~kb} / \mathrm{s})$. From Figure 3 through 9, sub-figure (a) and (b) compare the listening and conversational MOS, respectively.

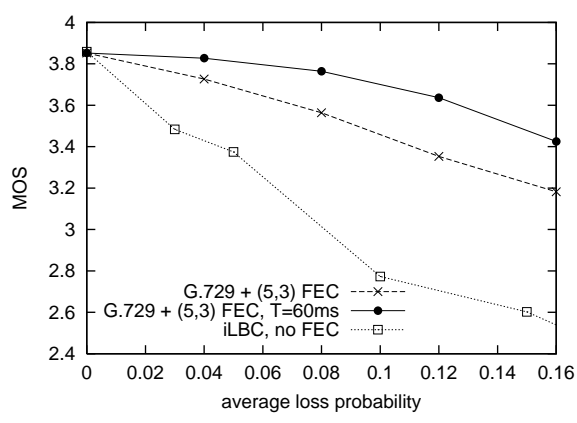

(a) ignoring delay effect

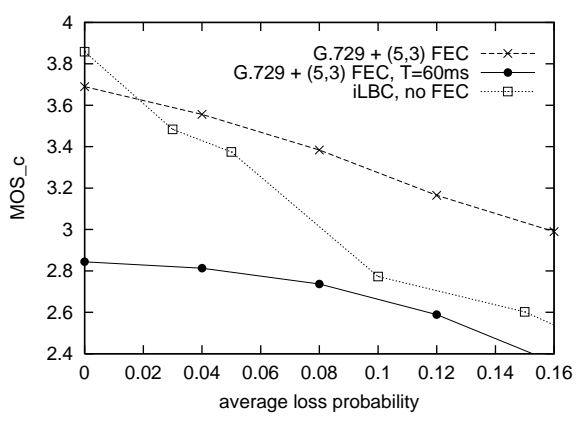

(b) considering delay effect

Figure 3. G.729 + (5,3) FEC code vs. iLBC without FEC; $T=30 \mathrm{~ms}$ by default

In Figure 3(a) MOS is highest for G.729 with a $60 \mathrm{~ms}$ interval, because FEC is more effective with a larger packet interval under bursty loss. ${ }^{9}$ Here both G.729 curves out-perform iLBC. But once we consider delay impairment, iLBC then prevails for low loss $(<2 \%)$, as in Figure 3(b). The G.729 curve starts lower than iLBC, because its FEC delay $(5 \times 30=150 \mathrm{~ms})$ attributes to a small impairment. However, beyond $2 \%$ loss, G.729 becomes increasingly better than iLBC due to its low packet loss after FEC. Finally, G.729 at

${ }^{b}$ We did not choose GSM EFR, AMR FR, or G.728 because their bit-rates are close to or even higher than iLBC, making the comparison moot or uninteresting. 
$60 \mathrm{~ms}$ now performs quite poorly, because its FEC delay is too high $(5 \times 60$ $=300 \mathrm{~ms}$ ). In Figure 2(b) delay impairment rises drastically beyond $150 \mathrm{~ms}$. Consequently, the gain in quality by using a larger packet interval is usually not enough to offset the excessive delay impairment. Therefore a rule of thumb is to keep FEC delay within $150 \mathrm{~ms}$, a requirement for an average interactive conversation. If a task or conversation is highly interactive, a lower threshold such as $100 \mathrm{~ms}$ may be used, and all we need to do is to adjust the delay impairment mapping in Figure 2(b) accordingly.

Our first result indicates G.729 with FEC is better than iLBC for a similar gross bit-rate, except when delay is considered and loss is low $(<2 \%)$.

\subsection{Adding FEC to Both G.729 and iLBC, Keeping Same Gross Bit-rate}

If we can afford more than $14 \mathrm{~kb} / \mathrm{s}$, then iLBC may use FEC as well, as in Figure 4, where iLBC uses a $(3,2)$ code, resulting in a $21 \mathrm{~kb} / \mathrm{s}$ gross bit-rate. G.729 uses a $(5,2)$ code, giving a comparable $20 \mathrm{~kb} / \mathrm{s}$.

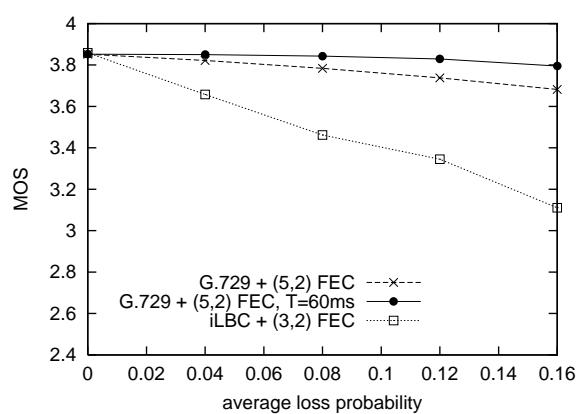

(a) ignoring delay effect

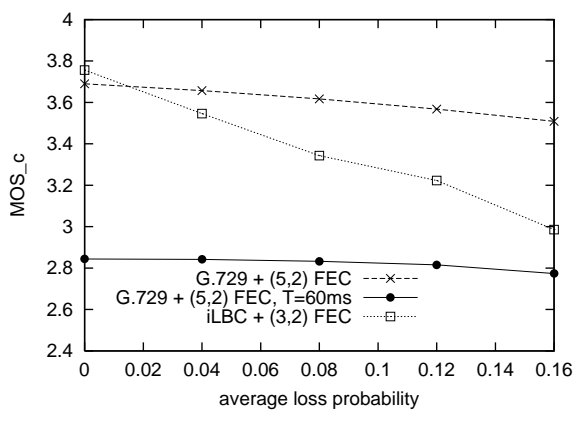

(b) considering delay effect

Figure 4. G.729 + (5,2) FEC code vs. iLBC $+(3,2)$ FEC code; $T=30 \mathrm{~ms}$ by default

In Figure 4(a), both G.729 curves are quite similar, because the FEC codes are already so redundant that virtually all losses are recovered. The quality of iLBC is much improved with FEC, but still lower than G.729. When considering delay, similar to Figure 3(b), iLBC is better when loss is low $(<2 \%)$, but G.729 prevails afterwards.

In Figure 5, iLBC employs a $(4,2)$ code, giving a $28 \mathrm{~kb} / \mathrm{s}$ gross bit-rate. G.729 uses a long $(7,2)$ code, resulting in $28 \mathrm{~kb} / \mathrm{s}$ as well. However, we do not show the G.729 $60 \mathrm{~ms}$ curve, because its difference with G.729 $30 \mathrm{~ms}$ curve is minimal when ignoring delay but too big otherwise. In Figure 5(a), iLBC still falls short of G.729. However, in Figure 5(b), iLBC is noticeably better 
than G.729 for loss rate as high as 14\%, because G.729's FEC delay is $7 \times 30$ $=210 \mathrm{~ms}, 60 \mathrm{~ms}$ larger than the $150 \mathrm{~ms}$ threshold in Figure 2(b). This is the case where iLBC dominates.

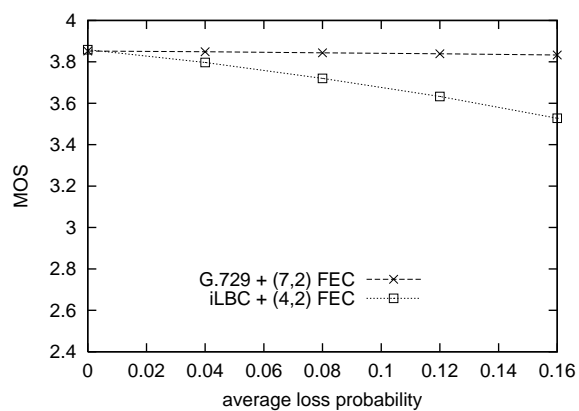

(a) ignoring delay effect

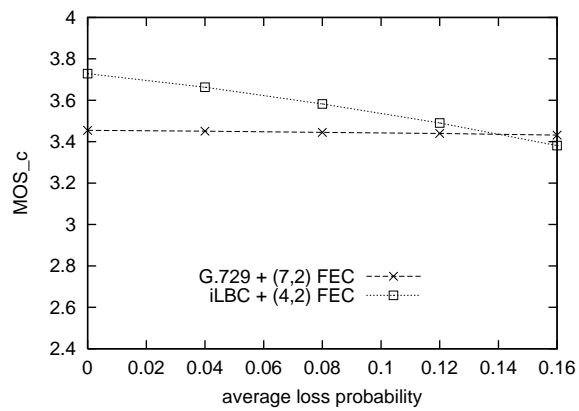

(b) considering delay effect

Figure 5. G.729 + (7,2) FEC code vs. iLBC $+(4,2)$ FEC code; $T=30 \mathrm{~ms}$ by default

The lower MOS of G.729 in Figure 5(b) is due to long FEC delay. By using a shorter FEC code, even with some concession in the bit-rate, higher conversational MOS may be possible. This is illustrated in Figure 6, where iLBC still uses a $(4,2)$ code, but G.729 changes to a $(3,1)$ code, using $24 \mathrm{~kb} / \mathrm{s}$ instead of $28 \mathrm{~kb} / \mathrm{s}$. The result: in both Figure 6(a) and (b), iLBC and G.729 perform very alike. Therefore sacrificing some FEC redundancy or using shorter FEC blocks may actually result in better conversational MOS.

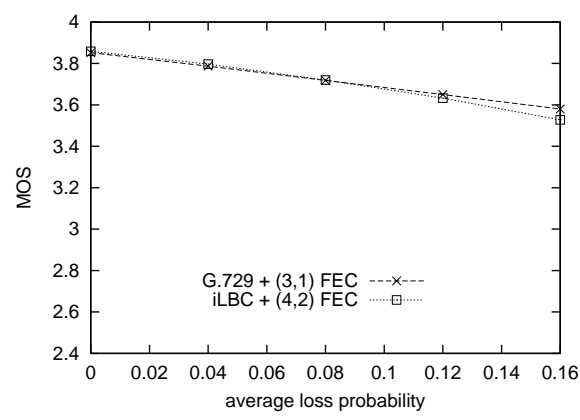

(a) ignoring delay effect

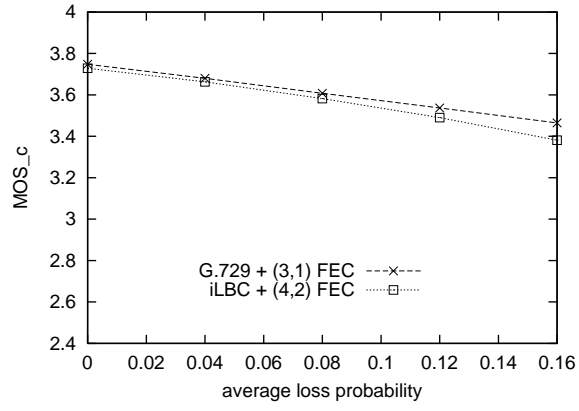

(b) considering delay effect

Figure 6. G. $729+(3,1)$ FEC code vs. iLBC $+(4,2)$ FEC code; $T=30 \mathrm{~ms}$ by default 


\subsection{Comparison with G.723.1}

Due to its lower bit-rate, G.723.1 has a lower intrinsic MOS at 0\% loss than G.729, but this also allows more FEC redundancy. In Figure 7, G.723.1 uses a $(2,1)$ FEC code $(12.6 \mathrm{~kb} / \mathrm{s})$, and iLBC without FEC. Similar to Figure 3(b), iLBC is better under low loss, but its dominant loss range is wider when considering delay. In Figure 7(b), G.723.1 performs better at $60 \mathrm{~ms}$ than at $30 \mathrm{~ms}$, because its FEC delay is only $2 \times 60=120 \mathrm{~ms}<150 \mathrm{~ms}$ threshold.

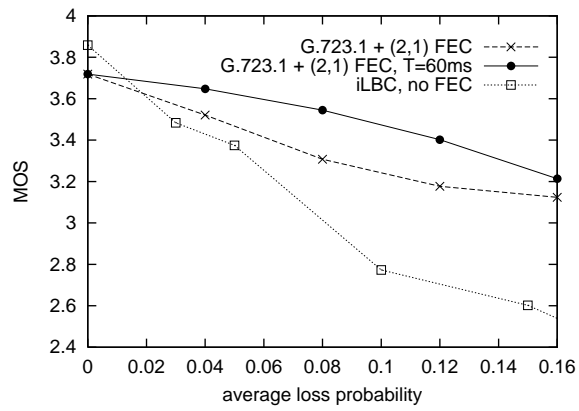

(a) ignoring delay effect

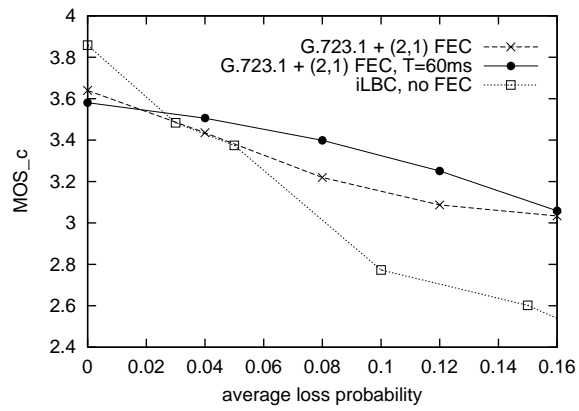

(b) considering delay effect

Figure 7. G.723.1 + (2,1) FEC code vs. iLBC without FEC; $T=30 \mathrm{~ms}$ by default

Figure 8 compares iLBC with a $(3,2)$ code $(21 \mathrm{~kb} / \mathrm{s})$ and $\mathrm{G} .723 .1$ with a $(3,1)$ code $(18.9 \mathrm{~kb} / \mathrm{s})$. Again, iLBC is better for low loss $(<3-6 \%)$.

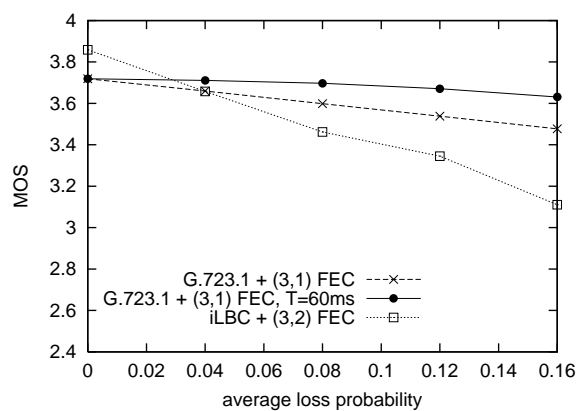

(a) ignoring delay effect

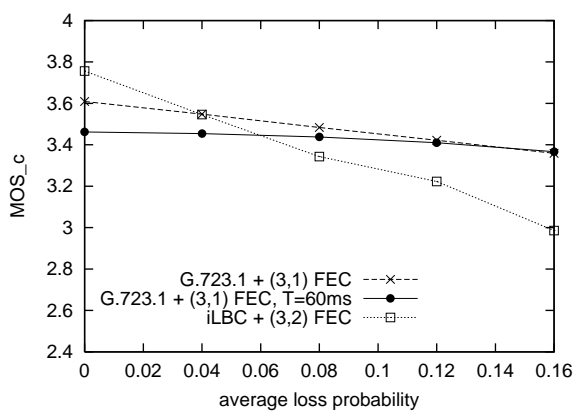

(b) considering delay effect

Figure 8. G.723.1 + (3,1) FEC code vs. iLBC $+(3,2)$ FEC code; $T=30 \mathrm{~ms}$ by default

Finally, Figure 9 compares iLBC with $(4,2)$ code $(28 \mathrm{~kb} / \mathrm{s})$ and G.723.1 with $(4,1)$ code $(25.2 \mathrm{~kb} / \mathrm{s})$. In both Figure $9(\mathrm{a})$ and (b), iLBC is the best for at least up to $8 \%$ loss, because G.723.1 has a lower intrinsic MOS.

icn 2002: submitted to World Scientific on June 5, 2002 


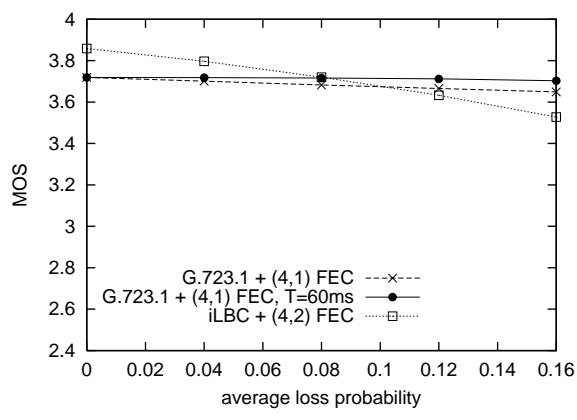

(a) ignoring delay effect

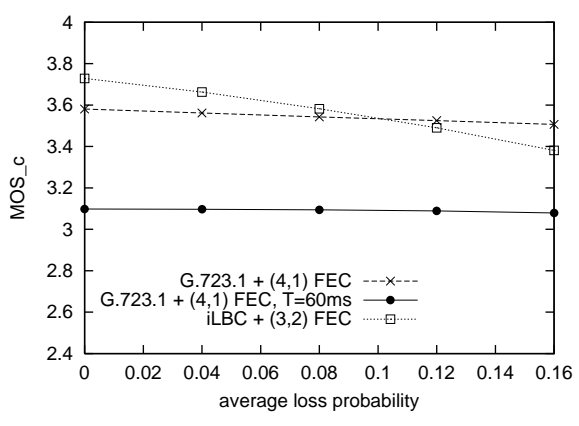

(b) considering delay effect

Figure 9. G.723.1 + (4,1) FEC code vs. iLBC + (4,2) FEC code; $T=30$ ms by default

\section{Discussion: The Best of Both Worlds}

In section 3, a common observation when considering delay is that iLBC prevails under low loss, but G.729 or G.723.1 is better for medium to high loss. Since none of them dominate solely, it is only beneficial to unite the best of both schemes. Therefore, we compute for a particular target bit-rate the maximum MOS these codecs can achieve. We consider conversational MOS $\left(M O S_{c}\right)$ only, as it is realistic to VoIP.

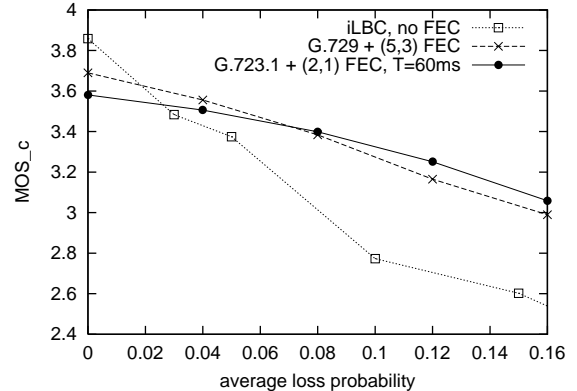

(a) The dominant MOS curves

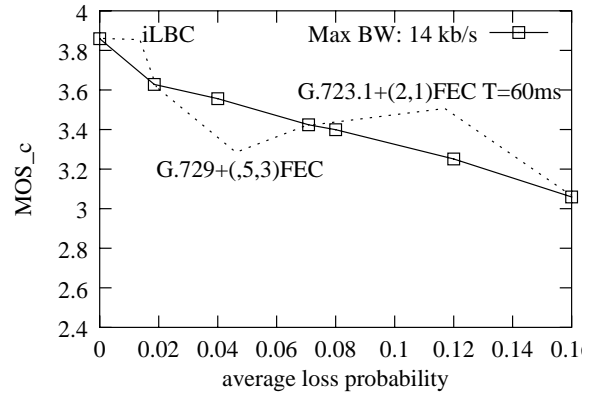

(b) Maximum of the dominant curves

Figure 10. Maximum conversational MOS achievable within $14 \mathrm{~kb} / \mathrm{s} ; T=30 \mathrm{~ms}$ by default

The first target bit-rate we evaluate is $14 \mathrm{~kb} / \mathrm{s}$. Based on Figure $3(\mathrm{~b})$ and 7(b), we find the highest $\operatorname{MOS}_{c}$ curves, $^{c}$ as shown in Figure 10(a). Then

${ }^{c}$ With a $10 \mathrm{~ms}$ frame, G.729 can use $40 \mathrm{~ms}, 50 \mathrm{~ms}$ or even $20 \mathrm{~ms}$ packet intervals too, but these cases turn out not to correspond to the dominant curves

icn 2002: submitted to World Scientific on June 5, 2002 
we take their maximum and arrive at the curve in Figure 10(b). This max $M O S_{c}$ curve has three segments, the first portion (low loss) corresponds to iLBC without FEC, the second part (medium loss) corresponds to G.729 + $(5,3)$ FEC. The last segment (high loss) is dominated by G.723.1 + $(2,1)$ FEC with a 60 ms packet interval, because G.723.1's lower bit-rate allows more FEC redundancy and its FEC delay $(120 \mathrm{~ms})$ is not too high.

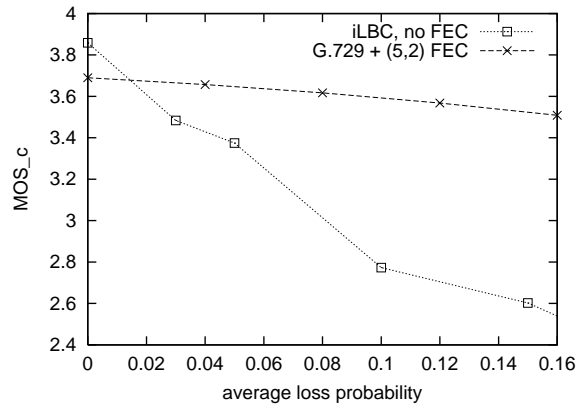

(a) The dominant MOS curves

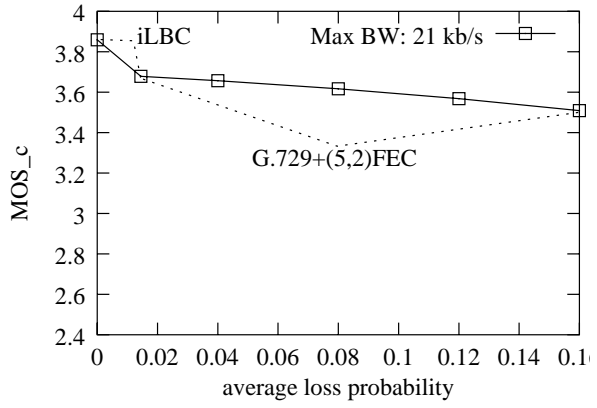

(b) Maximum of the dominant curves

Figure 11. Maximum conversational MOS achievable within $21 \mathrm{~kb} / \mathrm{s} ; T=30 \mathrm{~ms}$ by default

Figure 11 and 12 demonstrate the results for higher target bit-rates, $21 \mathrm{~kb} / \mathrm{s}$ and $28 \mathrm{~kb} / \mathrm{s}$, respectively. Figure 11(b) has two segments, the first one (low loss) again corresponds to iLBC with no FEC, and the second is G.729 + (5,2) FEC. G.723.1 + (3,1) FEC is also within $21 \mathrm{~kb} / \mathrm{s}$, but its MOS curve is lower than G.729 + $(5,2)$ FEC, therefore not listed for brevity.

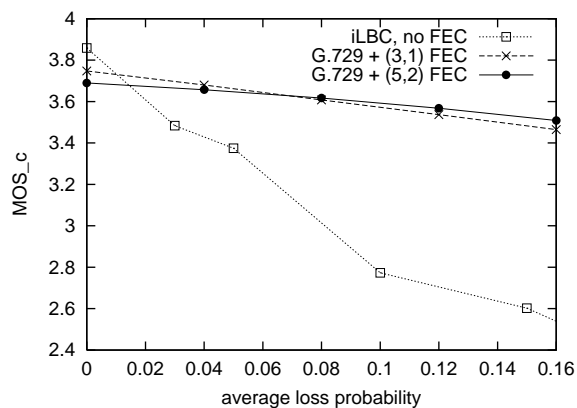

(a) The dominant MOS curves

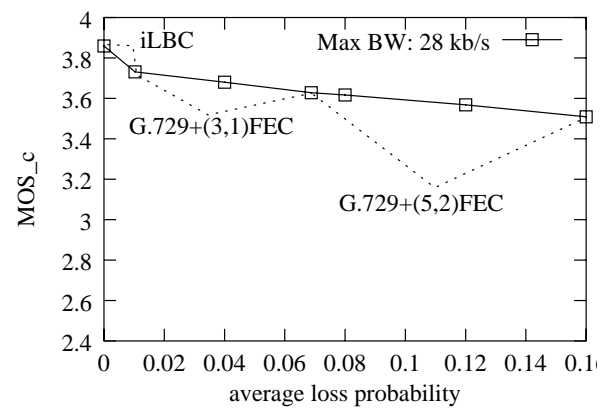

(b) Maximum of the dominant curves

Figure 12. Maximum conversational MOS achievable within $28 \mathrm{~kb} / \mathrm{s} ; T=30 \mathrm{~ms}$ by default 
Figure 12(b) has three segments. The first one (low loss) again belongs to iLBC without FEC, the second part is G.729 + (3,1) FEC. The third segment is G.729 + $(5,2)$ FEC, the same as in Figure 11(b). Although a $(3,1)$ code has more redundancy, its shorter block can sustain only $3-1=2$ consecutive losses. A $(5,2)$ code can sustain $5-2=3$ consecutive losses and its FEC delay is within the $150 \mathrm{~ms}$ threshold in Figure 2(b).

Finally, we plot in Figure 13 a comparison of Figure 10(b), 11(b), and 12(b). Figure 13 demonstrates the quality realizable for a certain target bitrate with the current state-of-the-art VoIP technology. The gain in $M O S_{c}$ is quite big from $14 \mathrm{~kb} / \mathrm{s}$ to $21 \mathrm{~kb} / \mathrm{s}$, but the improvement is marginal from $21 \mathrm{~kb} / \mathrm{s}$ to $28 \mathrm{~kb} / \mathrm{s}$. Although a higher target bit-rate allows more FEC redundancy, due to FEC delay impairment, there is an inherent limit to $M O S_{c}$.

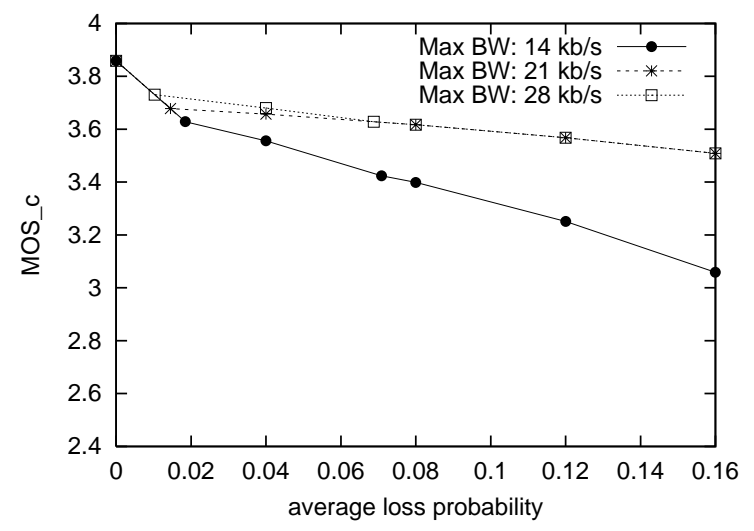

Figure 13. Effect of target bit-rate on maximum achievable conversational MOS

\section{Related Work}

Bolot ${ }^{13}$ discusses adaptation of FEC to maximize quality. However, the FEC used there is really low bit-rate redundancy (LBR), ${ }^{14}$ which is less general than FEC and it cannot recover lost audio with $100 \%$ accuracy. The perceived quality of LBR is also lower than FEC under the same gross bit-rate. ${ }^{9}$ The utility functions used by Bolot are hypothetical and do not correspond to real quality measures, whereas our results are based on real MOS curves. Finally we evaluate the effect of both loss and delay, instead of just loss.

We are not aware of other work that addresses the trade off between robust codec design and FEC or that derives the maximum attainable perceived quality with respect to packet loss within a target bit-rate.

icn 2002: submitted to World Scientific on June 5, 2002 


\section{Conclusions and Future Work}

We evaluate the perceived quality of conventional vs. robust low bit-rate codecs under the same bandwidth requirement, using the Mean Opinion Score (MOS) metric. When ignoring delay, the conventional codec with FEC is generally better. When considering delay impairment, the robust codec (iLBC) is better under low loss conditions. In some cases with high FEC redundancy, iLBC can dominate under most loss conditions.

We compute for a given target bit-rate, the quality achievable using either FEC or robust codec. We find that from $14 \mathrm{~kb} / \mathrm{s}$ to $21 \mathrm{~kb} / \mathrm{s}$ target bit-rate, there is a significant quality improvement. Yet from $21 \mathrm{~kb} / \mathrm{s}$ to $28 \mathrm{~kb} / \mathrm{s}$, the gain is marginal especially beyond $1 \%$ packet loss, limited inherently by increasing delay impairment introduced by FEC. Such limitation is unavoidable unless multiple communication paths are available, albeit at the expense of sending more packets and higher packet header overhead. Our evaluation reveals the quality realizable with the current state-of-the-art VoIP technology.

MOS results are subjective and require listening tests. However, the MOS performance of a given codec need only be measured once, and the remaining prediction of FEC MOS is done analytically, as outlined in Section 2. Our near-term plan is to implement the MOS prediction procedure in a VoIP application for real-time decision support on network adaptation. Another plan is to evaluate the effect of jitter on VoIP quality. Despite being effective against loss, FEC cannot reduce jitter unless out-of-order packets are common in the Internet. In contrast, packet loss concealment in the robust codec incurs a much lower delay. So we conjecture that a robust codec is more useful for battling jitter.

\section{Acknowledgments}

We thank Alan Duric at Global IP Sound for providing us MOS performance charts of iLBC and other codecs.

\section{References}

1. J. Rosenberg and H. Schulzrinne. An RTP payload format for generic forward error correction. RFC 2733, Internet Engineering Task Force, December 1999.

2. L. Rizzo. Effective erasure codes for reliable computer communication protocols. ACM Computer Communication Review, 27(2):24-36, April 1997.

icn 2002: submitted to World Scientific on June 5, 2002 
3. S. Andersen et al. Internet low bit rate codec. Internet Draft, Internet Engineering Task Force, February 2002. Work in progress.

4. International Telecommunication Union. Subjective performance assessment of telephone-band and wideband digital codecs. Recommendation P.830, Telecommunication Standardization Sector of ITU, Geneva, Switzerland, February 1996.

5. International Telecommunication Union. Coding of speech at $8 \mathrm{kbit} / \mathrm{s}$ using conjugate-structure algebraic-code-excited linear-prediction. Recommendation G.729, Telecommunication Standardization Sector of ITU, Geneva, Switzerland, March 1996.

6. International Telecommunication Union. Dual rate speech coder for multimedia communications transmitting at 5.3 and $6.3 \mathrm{kbit} / \mathrm{s}$. Recommendation G.723.1, Telecommunication Standardization Sector of ITU, Geneva, Switzerland, March 1996.

7. Soren Andersen and Alan Duric. IETF AVT WG draft-andersenilbc-00. Presentation, Global IP Sound, March 2002. Available at http://www.ietf.org/proceedings/02mar/slides/avt-6.pdf.

8. H. Schulzrinne, S. Casner, R. Frederick, and V. Jacobson. RTP: a transport protocol for real-time applications. RFC 1889, Internet Engineering Task Force, January 1996.

9. Wenyu Jiang and Henning Schulzrinne. Comparison and optimization of packet loss repair methods on voip perceived quality under bursty loss. In Proc. International Workshop on Network and Operating System Support for Digital Audio and Video (NOSSDAV), May 2002.

10. International Telecommunication Union. Application of the e-model: A planning guide. Recommendation G.108, Telecommunication Standardization Sector of ITU, Geneva, Switzerland, September 1999.

11. R. G. Cole and J. Rosenbluth. Voice over IP performance monitoring. ACM Computer Communication Review, 4(3), 2001.

12. Maya Yajnik, Sue Moon, Jim Kurose, and Don Towsley. Measurement and modelling of the temporal dependence in packet loss. In Proceedings of the Conference on Computer Communications (IEEE Infocom), New York, March 1999.

13. Jean-Chrysostome Bolot, Sacha Fosse-Parisis, and Don Towsley. Adaptive FEC-Based error control for interactive audio in the Internet. In Proceedings of the Conference on Computer Communications (IEEE Infocom), New York, March 1999.

14. Vicky Hardman, Angela Sasse, Mark Handley, and Anna Watson. Reliable audio for use over the Internet. In Proc. of INET'95, Honolulu, Hawaii, June 1995. 